\title{
Follicular adenoma of the thyroid gland in children: a clinical spectrum
}

\author{
Sylwester Gerus, Marcin Rasiewicz, Maciej Baglaj
}

Department of Pediatric Surgery and Urology, Wroclaw Medical University, Wroclaw, Poland

Submitted: 9 May 2020

Accepted: 29 November 2020

Arch Med Sci

DOI: https://doi.org/10.5114/aoms/130994

Copyright @ 2021 Termedia \& Banach

\section{Abstract}

Introduction: Surgical diseases of the thyroid gland in children are rare in comparison with those in the adult population. The aim of the study was to assess clinical aspects of thyroid follicular adenoma in children and attempt to develop a management algorithm that is useful in clinical practice.

Material and methods: The clinical database of all children operated on due to thyroid disease in the years 1993-2018 at the university pediatric surgical center was reviewed. The children in whom the postoperative histology report showed follicular adenoma were selected and their medical files were analyzed in detail. Clinical course of the disease, medical and family history, results of imaging studies, details of surgical management and final outcome were assessed in each case.

Results: Among 183 children operated on for thyroid pathology in the study period there were 56 patients with follicular adenoma (30.6\%). Their age ranged from 7 to 18 years. Seven patients were found to be hypothyroid and a further seven showed hyperthyroid status initially. Ultrasound scan showed a solitary nodule in 37 children (66.1\%). Multiple nodules in one lobe were detected in 5 patients, while a further 14 children showed bilateral thyroid pathology. The result of preoperative cytologic examination was suspicious in 12 children (21.4\%). All children were subjected to operative management. Forty-two children (75\%) underwent at least unilateral total lobectomy. No patient showed a recurrent thyroid disease.

Conclusions: Follicular adenoma in children presents a wide clinical and morphological spectrum. High incidence of associated diseases of other systems and congenital anomalies of the thyroid gland may indicate a complex etiology of follicular adenoma with participation of still unknown endogenic factors in children.

Key words: children, lobectomy, benign tumor, fine-needle biopsy.

\section{Introduction}

Surgical diseases of the thyroid gland in children are rare in comparison with those in the adult population $[1,2]$. They evoke interest due to a relatively high incidence of malignant tumors, which varies between 15 and $20 \%$ of the excised thyroid nodules [1-3]. Although benign tumors of the thyroid gland constitute a major part of surgical thyroid pathology in children, the medical literature about these tumors is scarce. Benign tumors are discussed collectively with malignant lesions by most authors, which obscures their clinical features. The most common benign lesion of the thyroid gland is follicular adenoma, which constitutes 33-75\%

\author{
Corresponding author: \\ Sylwester Gerus \\ Department of Pediatric \\ Surgery and Urology \\ Wroclaw Medical University \\ 213 Borowska St \\ 50-556 Wroclaw, Poland \\ E-mail: \\ sylwester.gerus@umed.wroc.pl
}


of the nodular lesions. Taking into account the rising incidence of follicular adenoma in the last decade in Lower Silesia (south-western part of Poland) the authors undertook a retrospective study that aimed to assess clinicopathological aspects of thyroid follicular adenoma in children.

\section{Material and methods}

Retrospective assessment of the medical files of children operated on due to thyroid disease at the Pediatric Surgery and Urology Clinic at the Medical University in Wroclaw between 1993 and 2018 was undertaken. Only patients in whom the postoperative pathological report revealed follicular adenoma were included in the study. The following data were analyzed: demographic data, family history, comorbid conditions and diseases, the results of preoperative diagnostic laboratory and imaging studies, intraoperative findings, mode of surgical resection and postoperative complications.

\section{Results}

This was a retrospective observational study and the current study was approved by the Clinical Research Ethic Committee of Wroclaw Medical
University (number KB-175/2010). Since this study has a retrospective design, no informed consent was required.

Among 183 children operated on due to thyroid diseases, follicular adenoma was finally diagnosed in 56 (30.6\%) of them. The patients were between 7 and 18 years of age (the mean age was 14.3). The majority of the patients were girls and the ratio of girls to boys was 2.5: 1 (40 girls to 16 boys). In 15 cases, family history was positive (in 9 girls and 6 boys). In 8 cases, more than one family member had been diagnosed with thyroid disease. Twelve children (12.4\%) were diagnosed with comorbid conditions and diseases (Table I).

In 42 (75\%) cases hormonal levels were normal, while 7 children (12.5\%) were diagnosed with hyperthyroidism and 7 children were treated for hypothyroidism (12.5\%). Thyroid ultrasonographic examinations (US) showed lesions in one lobe only in 42 cases (75\%) and in 14 patients (25\%) the lesions were bilateral. In the former group, a single lesion was found in 37 patients and multiple lesions in a further 5 patients. In the latter group, single lesions in both lobes were found in 6 cases, multiple lesions in both lobes in 3 cases and a single lesion in one lobe and multiple lesions in the contralateral lobe were found in

Table I. Patient demographics

\begin{tabular}{|c|c|c|c|}
\hline Parameter & Total & Female patients & Male patients \\
\hline Number of patients, $n(\%)$ & $56(100)$ & $40(71)$ & $16(29)$ \\
\hline Age [years], average \pm SD & $14.3 \pm 2.85$ & $14.75 \pm 2.85$ & $12.68 \pm 2.22$ \\
\hline Positive familial history, $n$ (\%) & $15(27)$ & $9(16)$ & $6(11)$ \\
\hline \multicolumn{4}{|l|}{ Hormonal status before operation, $n(\%)$} \\
\hline Euthyroid (TSH level 0.4-4 mU/l) & $42(75)$ & $29(52.0)$ & $13(23.0)$ \\
\hline Hypothyroid (TSH level: > 4 mU/l) & 7 (12.5) & $5(8.9)$ & $2(3.6)$ \\
\hline Hyperthyroid (TSH level: 0-0.4 mU/l) & $7(12.5)$ & $6(10.7)$ & $1(1.8)$ \\
\hline \multicolumn{4}{|l|}{ Comorbid conditions } \\
\hline Malignant neoplasms & 2 & Retinoblastoma & Wilms tumor \\
\hline Benign neoplasms & 2 & Ovarian teratoma & Angiofibrolipoma \\
\hline Polycystic kidney disease & 1 & 1 & - \\
\hline Hemosiderosis & 1 & - & 1 \\
\hline Pathological obesity & 1 & - & 1 \\
\hline Malformation of the thumb & 1 & - & 1 \\
\hline Epilepsy & 1 & 1 & - \\
\hline Hypoacusis & 1 & 1 & - \\
\hline$\alpha$-1-antitrypsin & 1 & - & 1 \\
\hline Tuberculosis & 1 & 1 & - \\
\hline
\end{tabular}

TSH - thyroid stimulating hormone, $\mathrm{mU} / \mathrm{l}$ - milliunits per liter, SD - standard deviation 


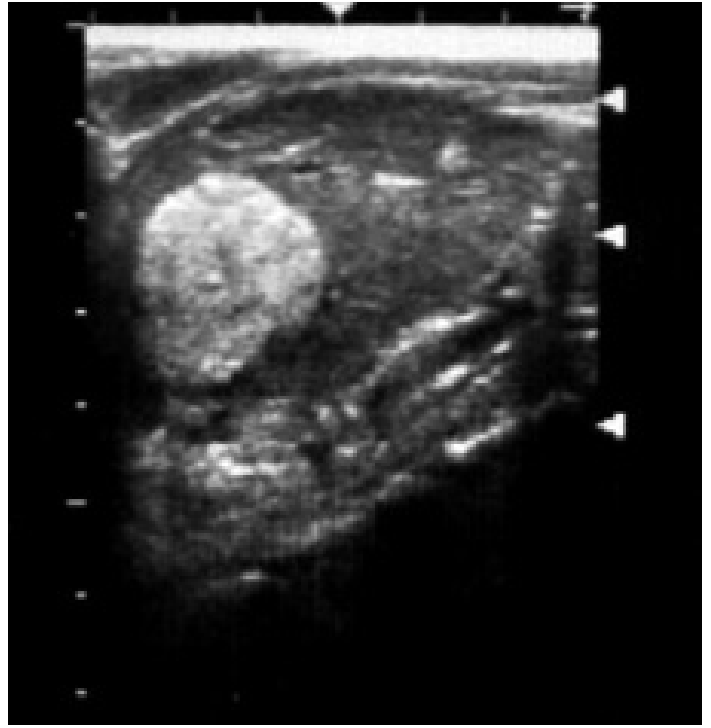

Figure 1. Ultrasonographic image of follicular adenoma - single lesion

5 patients. In 21 cases (37.5\%) single lesions had a solid structure and in 19 cases (33.9\%) both solid and cystic components were noted. Calcifications were found in 6 children. In 27 cases the size of a single focal lesion exceeded $25 \mathrm{~mm}$. Twenty children (35.7\%) underwent thyroid scintigraphy (Figures 1 and 2). A cold nodule was found in 7 cases, a hot nodule in 5 cases and an autonomous one in 2 cases (Figures 3 and 4). Cytological testing of samples taken by fine-needle biopsy (FNAB) under ultrasound control was performed in 53 cases. Twelve children (22.6\%) were diagnosed with a follicular lesion. In 3 children (5.7\%) suspicion of malignant tumor was raised. In 35 patients (66.1\%) cytological examination revealed a benign lesion. In 3 cases (5.6\%) the FNAB samples were non-diagnostic.

All 56 children underwent surgical treatment. The mode of thyroid resection was determined by the result of preoperative cytological examination, topography and dimensions of pathological lesions in the thyroid gland (Table II). Total removal of the affected thyroid lobe with preservation of the contralateral lobe or its partial resection was performed in 42 children (75\%). Two patients had both of their lobes totally removed. The first patient was a girl diagnosed with thyroid cancer suspected on preoperative cytological assessment. She showed symptoms of hypothyroidism and her family history was positive. The other patient was a boy with a positive family history and numerous nodules in both lobes. In 10 cases intraoperative assessment of the resected specimen was ordered and in all cases it showed a benign lesion (Figure 5).

In 19 children (34\%) the presence of one or more anatomical abnormalities of the thyroid gland was diagnosed intraoperatively (Table III).

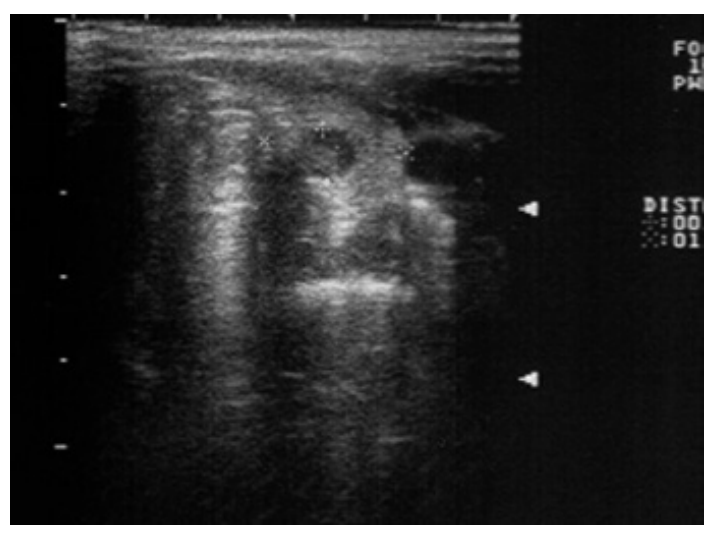

Figure 2. Ultrasonographic image of follicular adenoma - multiple lesions

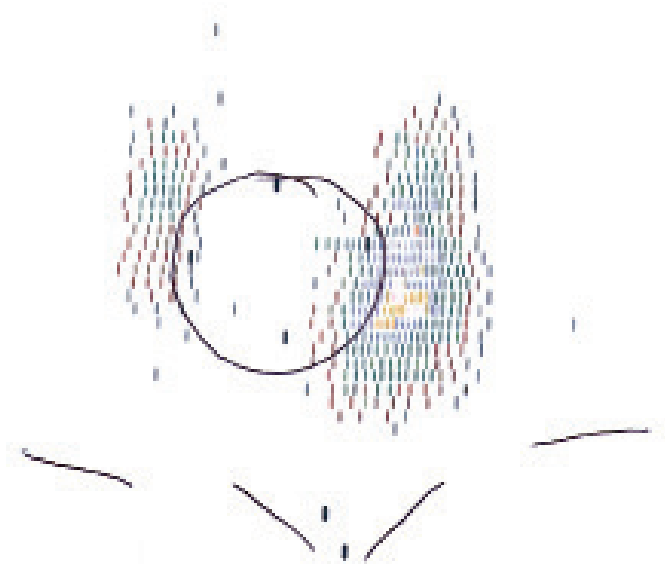

Figure 3. Scintigraphy - cold nodule

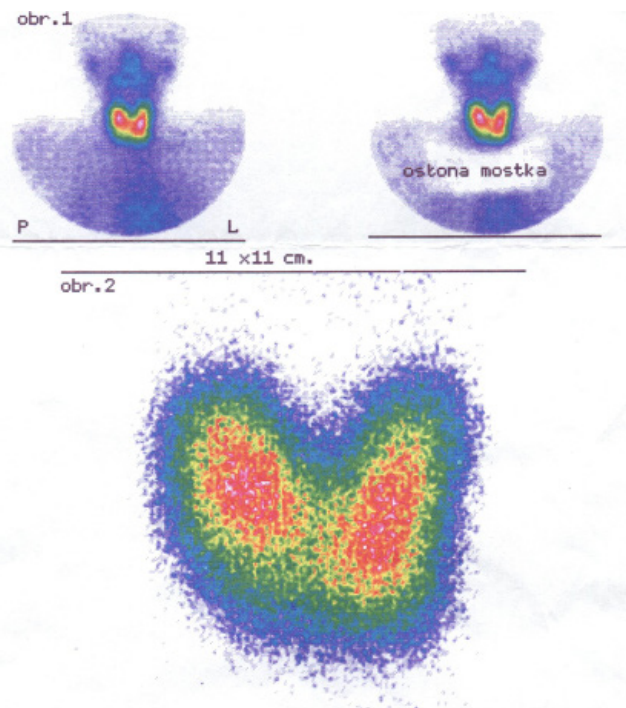

Figure 4. Scintigraphy - hot nodules

In most cases, there were no postoperative complications. One girl experienced postoperative bleeding, which required urgent wound revision. In two other cases, histopathological examinations showed the presence of a parathyroid gland. 
Table II. Type of thyroid resections (part - partial, sub - subtotal, tot - total lobectomy)

\begin{tabular}{|lc|}
\hline Procedure & $n(\%)$ \\
\hline tot/tot & $2(3.6)$ \\
\hline tot/sub & $6(10.7)$ \\
\hline tot/part & $4(7.2)$ \\
\hline tot/--- & $30(53.5)$ \\
\hline sub/sub & $5(8.9)$ \\
\hline sub/part & $6(10.7)$ \\
\hline sub/--- & $1(1.8)$ \\
\hline part/--- & $2(3.6)$ \\
\hline
\end{tabular}

Table III. Anatomical abnormalities of thyroid gland

\begin{tabular}{|l|c|}
\hline Abnormality & $n$ \\
\hline Pyramidal lobe & 9 \\
\hline Undescended thyroid gland & 9 \\
\hline Underdeveloped isthmus & 4 \\
\hline Thyroglossal duct & 2 \\
\hline L-shaped thyroid gland & 1 \\
\hline Underdeveloped lobe & 2 \\
\hline
\end{tabular}

In one patient a hypertrophic scar was noted at the postoperative follow-up. All patients remain under combined surgical and endocrinological follow-up review until 18 years of age (mean 5.3 years). No recurrence was noted.

\section{Discussion}

Nodular lesions of the thyroid gland in children are much less frequent than in adults and the majority of them are benign. Follicular adenoma is the most common benign lesion [2, 4]; however, only one article concerning this issue in children has been found in medical databases. The authors described 5 patients. These were 4 girls and 1 boy between the age of 11 months and 18 years. Hormonal levels were normal in all patients. Scintigraphic examinations showed cold nodules. One patient underwent a cytological examination. A total lobectomy was performed in 4 cases and a subtotal one in 1 case [2].

In the vast majority of publications, children with follicular adenoma are described together with children with other tumors of the thyroid gland, which obscures its true clinical features. In the Pediatric Surgery and Urology Clinic at the Medical University of Wroclaw, 183 children were operated on due to nodular thyroid diseases between 1993 and 2018. Fifty-six (30.6\%) were finally diagnosed with follicular adenoma. To
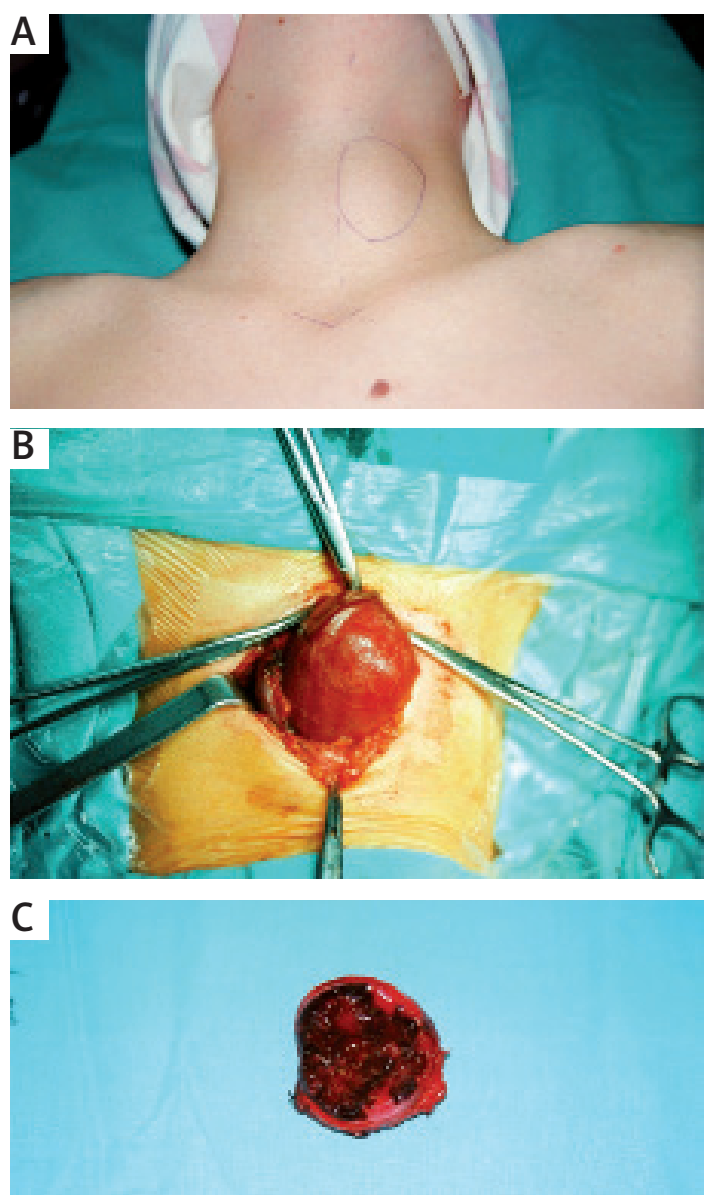

Figure 5. Preoperative, intraoperative and postoperative view of follicular adenoma

the authors' knowledge, this is the largest series of children with this benign tumor of the thyroid gland presented to date in the medical literature.

Follicular adenoma is a benign tumor that consists of follicular epithelial cells, differs from the surrounding glandular parenchyma and has a capsule [2, 5]. Relevant literature suggests that it occurs approximately twice as often in girls as in boys and the average age of patients is around 12.5 years $[2,6]$.

In our material, the ratio of girls to boys was approximately $2.5: 1$ and the patients were between 7 and 18 years of age. The average age of the patient was 14.3 years. Family history was positive in 15 patients $(26.8 \%)$. It is consistent with the data from the literature which state that thyroid diseases occur among thyroid patients' family members in $23.3 \%$ of cases. In one case, a nodular thyroid disease occurred in a patient's twin sister. Analyzing the demographic data and family history in the presented material, it seems that follicular adenoma follows the patterns of common nodular goiter in children.

It is however worth mentioning that children with follicular adenoma frequently present with 
medical history revealing associated conditions or diseases. Four of the presented patients were treated for malignant and benign neoplasm. Reviewing the database of all patients with thyroid nodular disease, we found that the incidence of comorbid conditions was significantly higher in the group of follicular adenoma than in children with other thyroid lesions. The paucity of data in the medical literature does not allow these data to be compared with the experience of other authors, but this issue without doubt requires further exploration.

Preoperative procedures in children with follicular adenoma do not differ substantially from those with other lesions in the thyroid gland. Currently in our center US of the thyroid gland, hormonal studies and FNAB are required preoperatively in all children with thyroid nodular disease. Since 2002 all thyroid biopsies have been performed under US guidance by two senior authors involved in surgical management of children with thyroid pathology.

The ultrasound examination currently plays a major role in imaging of nodular thyroid diseases in children. It is relatively inexpensive, safe, repeatable and allows for assessment of the structure and location of the gland and the surrounding lymph nodes as well as the morphology of the nodules. None of the ultrasound features is pathognomonic for a malignant tumor [7, 8]. An ultrasound image of a follicular adenoma is not characteristic either. Our patients were diagnosed with single and multiple lesions in one as well as both lobes. The nodules had a solid or cystic structure or consisted of both solid and cystic components. They also varied in echogenicity and size (from several $\mathrm{mm}$ to over $3 \mathrm{~cm}$ ). In a few patients, calcifications in the proximity of nodular lesions were discovered. The role of the scintigraphic examination has changed over the years. Thyroid scintigraphy was performed routinely until 2000, and since then it has been ordered only in selected cases with hyperthyroidism [7]. At present, the gold standard in diagnosis of nodular thyroid diseases is fine-needle biopsy conducted under ultrasound control [9]. In the presented material, it was performed in 53 children (95\% of the patients). Cytological examination does not allow for differentiation of benign follicular adenoma and malignant follicular carcinoma, although the latter is a very rare disease in the pediatric population. Thyroid biopsy in children is not an easy procedure and requires a team of an experienced radiologist, pediatric surgeon and pathologist $[10,11]$. Small thyroid lesions of diameter less than $1 \mathrm{~cm}$ pose a diagnostic challenge. In the majority of the children, the result of a cytological examination indicated a benign lesion. Diagnosis of follicular lesion on cytology was an indication for intraoperative assessment of the resected specimen of the thyroid gland until 2002. Since then we have abandoned intraoperative pathology as total unilateral lobe resection has been introduced as the method of choice of surgical management in all operated patients. Follicular carcinoma was noted only in 5 children throughout the study period.

The treatment of choice in the case of thyroid nodules in children is their surgical removal. The extent of resection is determined by the topography and dimensions of pathological changes in the gland. However, there are no uniform guidelines as to the extent of resection. It often depends on the experience of a particular medical center or even the surgeon performing the operation. In recent years, an increasing number of authors are recommending total removal of at least one lobe in children with nodular thyroid disease [7, 12, 13]. This choice is safe, characterized by a small percentage of complications and prevents recurrence of nodular diseases $[13,14]$. In our material, 42 patients (75\%) had at least one of their thyroid lobes removed. Analyzing the operations performed in the authors' center, important evolution of the surgical strategy in children with nodular thyroid diseases has been noted. Before 2002, the preferred type of surgery was a subtotal removal of a lobe following a pattern accepted in general surgery, whereas after 2002 it was a total resection of at least one lobe. The change in the operative approach primarily resulted from the necessity to operate again on 3 patients with malignant tumors in whom malignant cells had not been found in a cytological examination and who had undergone a subtotal removal of a thyroid lobe. The second reason was that nodular lesions, other than adeno$\mathrm{ma}$, recurred in the glandular tissue that remained after the incomplete removal of the lobe in 2 patients. Unilateral complete resection of the thyroid gland has become the minimal extent of surgical intervention in any child with nodular disease of the thyroid gland in the authors' center. In children with nodular disease and hypothyroidism total resection of the gland is recommended due to higher risk of recurrence and higher risk of intraoperative complications during the second surgical intervention.

An interesting observation is the presence of congenital defects of the thyroid gland detected intraoperatively in children with follicular adenoma. In our material, such defects were found in 19 children (34\%). This issue has not been discussed in the medical literature. Anatomical abnormalities in children with adenoma were noted significantly more often than in children with nodular goiter. This is another topic deserving an objective and prospective study.

Our study has several strengths: this is only the second report about follicular adenoma and 


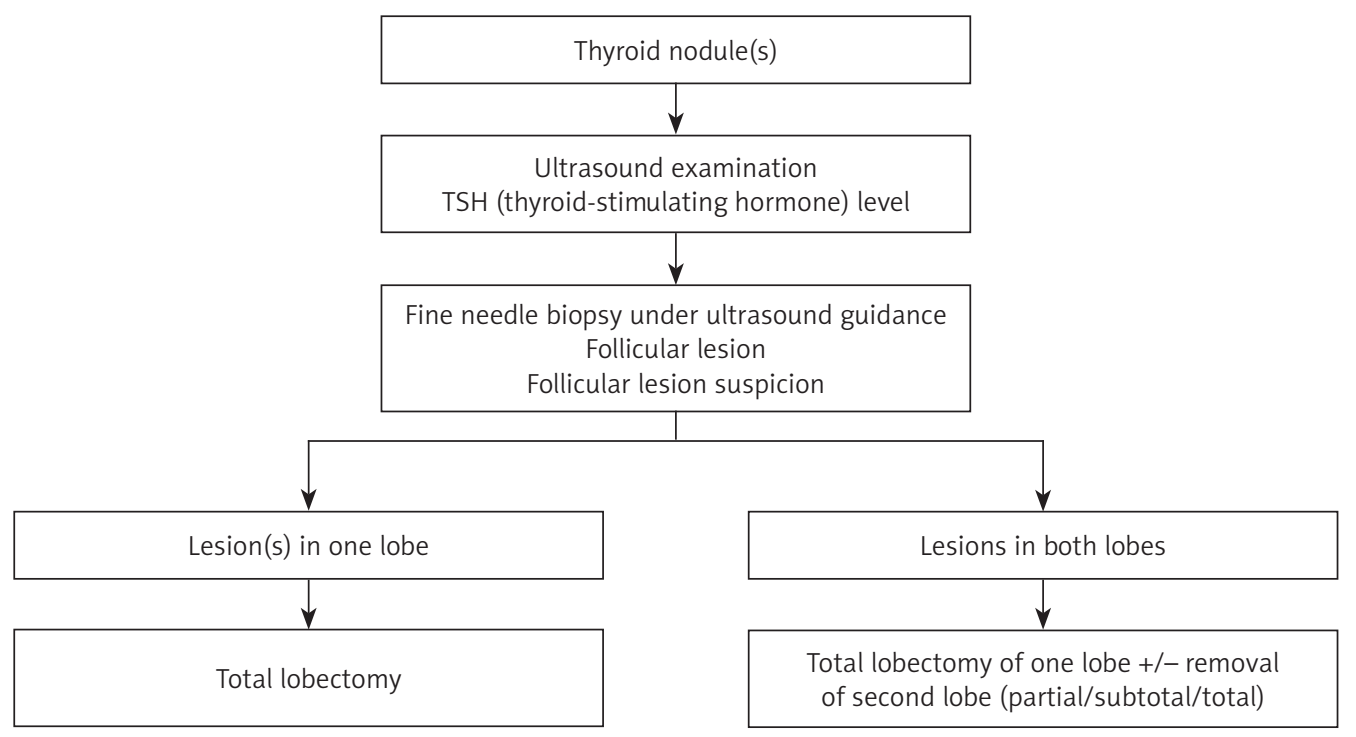

Figure 6. Proposed algorithm for management of a patient with follicular adenoma

contains the largest number of patients. It is from a single center and the patients were operated on by one surgical team consecutively. We acknowledge that this study also has limitations: a single center, and lack of genetic studies.

Proposed algorithm for management of a patient with follicular adenoma see Figure 6.

In conclusion, management of a child with a follicular adenoma follows the pattern accepted for children with nodular thyroid disease. Follicular adenoma may present as a unilateral or bilateral thyroid disease affecting one or two lobes. There is no pathognomonic clinical or US pattern of follicular adenoma in children. Fine needle biopsy plays a pivotal role in preoperative diagnosis of the thyroid pathology. A high rate of associated conditions and anatomic abnormalities of the thyroid gland may indicate a complex etiologic background of follicular adenomas accompanied by unknown endogenous factors. Active treatment of follicular adenoma in children is the most appropriate management.

\section{Conflict of interest}

The authors declare no conflict of interest.

\section{References}

1. Ledbetter DJ. Thyroid surgery in children. Semin Pediatr Surg 2014; 23: 60-5.

2. Festen C, Otten BJ, van de Kaa CA. Follicular adenoma of the thyroid gland in children. Eur J Pediatr Surg 1995; 5: 262-4.

3. Hung W, Anderson KD, Chandra RS, et al. Solitary thyroid nodules in 71 children and adolescents. J Pediatr Surg 1992; 27: 1407-9.

4. Mirshemirani A, Rozhanzamir F, Tabari AK, et al. Thyroid nodules in childhood: a single institute experience. Iran J Pediatr 2010; 20: 91-6.
5. Meissner WA. Follicular carcinoma of the thyroid. Am J Surg Pathol 1977; 1: 171-3.

6. Angerpointner TA, Britsch E, Knorr D, et al. Surgery for benign and malignant diseases of the thyroid gland in childhood. Prog Pediatr Surg 1991; 26: 21-7.

7. Baglaj M, Gerus S, Dorobisz U, et al. Thyroidectomy in children: changing trends and surgical strategies. Adv Clin Exp Med 2013; 22: 387-93.

8. Yoskovitsch A, Laberge JM, Rodd C, et al. Cystic thyroid lesions in children. J Pediatr Surg 1998; 33: 866-70.

9. Scholz S, Smith JR, Chaignaud B, Shamberger RC, Huang SA. Thyroid surgery at Children's Hospital Boston: a 35-year single-institution experience. J Pediatr Surg 2011; 46: 437-42.

10. Lugo-Vicente H, Ortiz VN. Pediatric thyroid nodules: insights in management. Bol Asoc Med P R 1998; 90: 74-8.

11. Corrias A, Mussa A. Thyroid nodules in pediatrics: which ones can be left alone, which ones must be investigated, when and how. J Clin Res Pediatr Endocrinol 2013; 5 (Suppl 1): 57-69.

12. Breuer C, Tuggle C, Solomon D, et al. Pediatric thyroid disease: when is surgery necessary, and who should be operating on our children? J Clin Res Pediatr Endocrinol 2013; 5 (Suppl 1): 79-85.

13. Burke JF, Sippel RS, Chen H. Evolution of pediatric thyroid surgery at a tertiary medical center. J Surg Res 2012; 177: 268-274.

14. Raval MV, Browne M, Chin AC, et al. Total thyroidectomy for benign disease in the pediatric patient - feasible and safe. J Pediatr Surg 2009; 44: 1529-33. 